\title{
SIRTF science operations system
}

\section{William B. Green}

William B. Green, "SIRTF science operations system," Proc. SPIE 4850, IR Space Telescopes and Instruments, (5 March 2003); doi: 10.1117/12.461105 Hawai'i, United States 


\title{
The SIRTF Science Operations System
}

\author{
William B. Green and the Staff of the SIRTF Science Center \\ SIRTF Science Center \\ California Institute of Technology \\ Pasadena, CA
}

\begin{abstract}
The Space Infrared Telescope Facility (SIRTF) will be launched in early 2003, and will perform an extended series of science observations at wavelengths ranging from 3.6 to 160 microns for five years or more. The California Institute of Technology has been selected as the home for the SIRTF Science Center (SSC). The SSC is completing the final stages of prelaunch development and testing of the Science Operations System (SOS), which will support science operations of the Observatory. The SOS supports a variety of functions including observing proposal submission by the scientific community, long range planning and short term scheduling of the Observatory, instrument performance monitoring during nominal operations, and production of a variety of scientific archival products. This paper describes the role and function of the SSC, the architecture of the SOS, and discusses the major SOS subsystems. Examples of products generated by the SOS are included.
\end{abstract}

\section{SIRTF SCIENCE CENTER ROLE AND FUNCTION}

The Space Infrared Telescope Facility (SIRTF) will be launched in early 2003, and will perform an extended series of science observations at wavelengths ranging from 3.6 to 160 microns for five years or more. The California Institute of Technology has been selected as the home for the SIRTF Science Center (SSC). The SSC is responsible for evaluating and selecting observation proposals, providing technical support to the science community, performing mission planning and science observation scheduling activities, instrument calibration during operations and instrument performance monitoring, production of archival quality data products, and management of science research activities. The SSC is responsible for design, development, and operation of the Science Operations System (SOS) which will support the functions assigned to the SSC by NASA.

The activities supported by the SSC are shown in Figure 1. In order to allocate observing time on the Observatory, the SSC solicits observing proposals from the Science community, administers proposal evaluation using a Time Allocation Committee structure, selects proposals, and administers funding of selected General Observers (GO's). Observation proposals are submitted using SSC software that expands observing requests to the command level and provides feedback to the users on utilization of Observatory resources for each observation. In addition to General Observers, two other Observer communities have been allocated observing time on the Observatory. The Guaranteed Time Observers (GTO's) include the Principal Investigator (PI) teams that have been responsible for development of the three payload instruments and other members of the Project Science Working Group. The SIRTF Legacy Teams are six teams that were selected through a competitive process managed by the SSC. Each of the six teams will conduct a specific observation campaign early during flight operations, and will release data products to the scientific community through the SSC as early as possible during operations to provide a basic set of archival science data to the community at an early date. The SSC integrates selected GO observation requests with observations requested by the GTO's and the SIRTF Legacy Programs to generate a long term observation schedule and detailed weekly sequence products. These are delivered to the Jet Propulsion Laboratory (JPL) Mission Operations organization for uplink to the Observatory. 


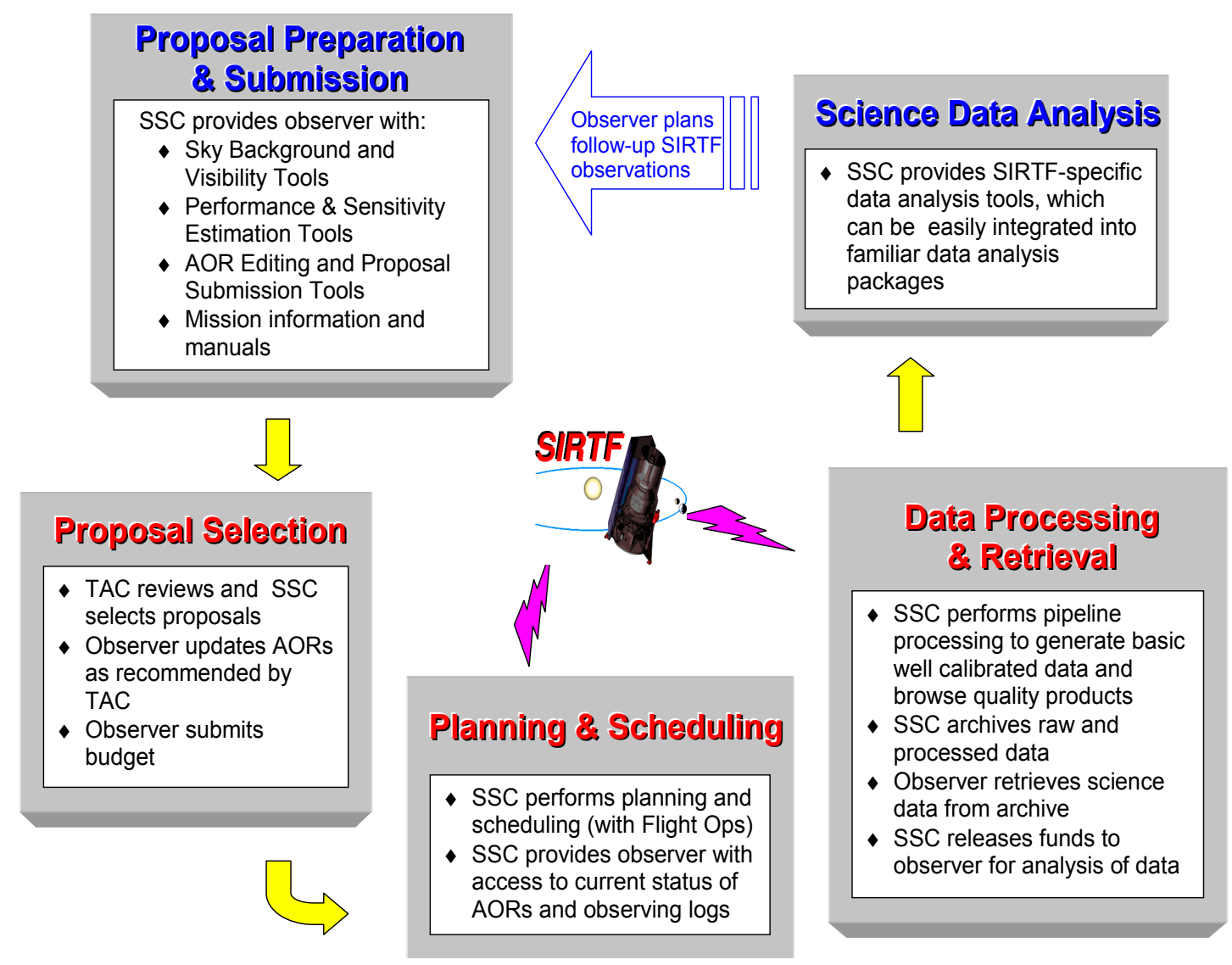

Fig. 1:Basic Functions Supported by the SIRTF Science Center

Following successful execution of the observations, the data are downlinked from the Observatory to the Mission Operations System (MOS) at JPL. Science and engineering data are packaged by JPL and transferred to the SSC. The SSC becomes responsible for long term instrument performance analysis following the In Orbit Checkout phase of the mission, and has responsibility for production of archival data products.

\section{SCIENCE OPERATIONS SYSTEM OVERVIEW}

The key requirements on the Science Operations System (SOS) are shown in Table 1. The SOS design includes modules for each of the major functions supported by the SSC. A block diagram of the system is shown in Figure 2.

\begin{tabular}{|cl|}
\hline & \multicolumn{1}{c|}{ Table 1 } \\
Science Operations System Driving Requirements \\
\hline - & Solicit, evaluate and select from $\sim 1200$ proposals per cycle \\
\hline$\bullet$ & Support observation planning and scheduling for $\sim 400$ programs per cycle \\
\hline - & Schedule $~ 20,000$ approved Astronomical Observing Requests per year \\
\hline - & Process and calibrate 12 hours worth of science data within 6 hours of receipt \\
\hline - & Support in-orbit checkout and science verification of instruments and observation modes \\
\hline - & Assess and monitor scientific performance of the instruments \\
\hline - & Develop and implement calibration plans and verify their outcome \\
\hline - & Manage all aspects of data analysis funding for approved observational and archival research \\
\hline - & Support community access to data products, data analysis and archival research results \\
\hline
\end{tabular}




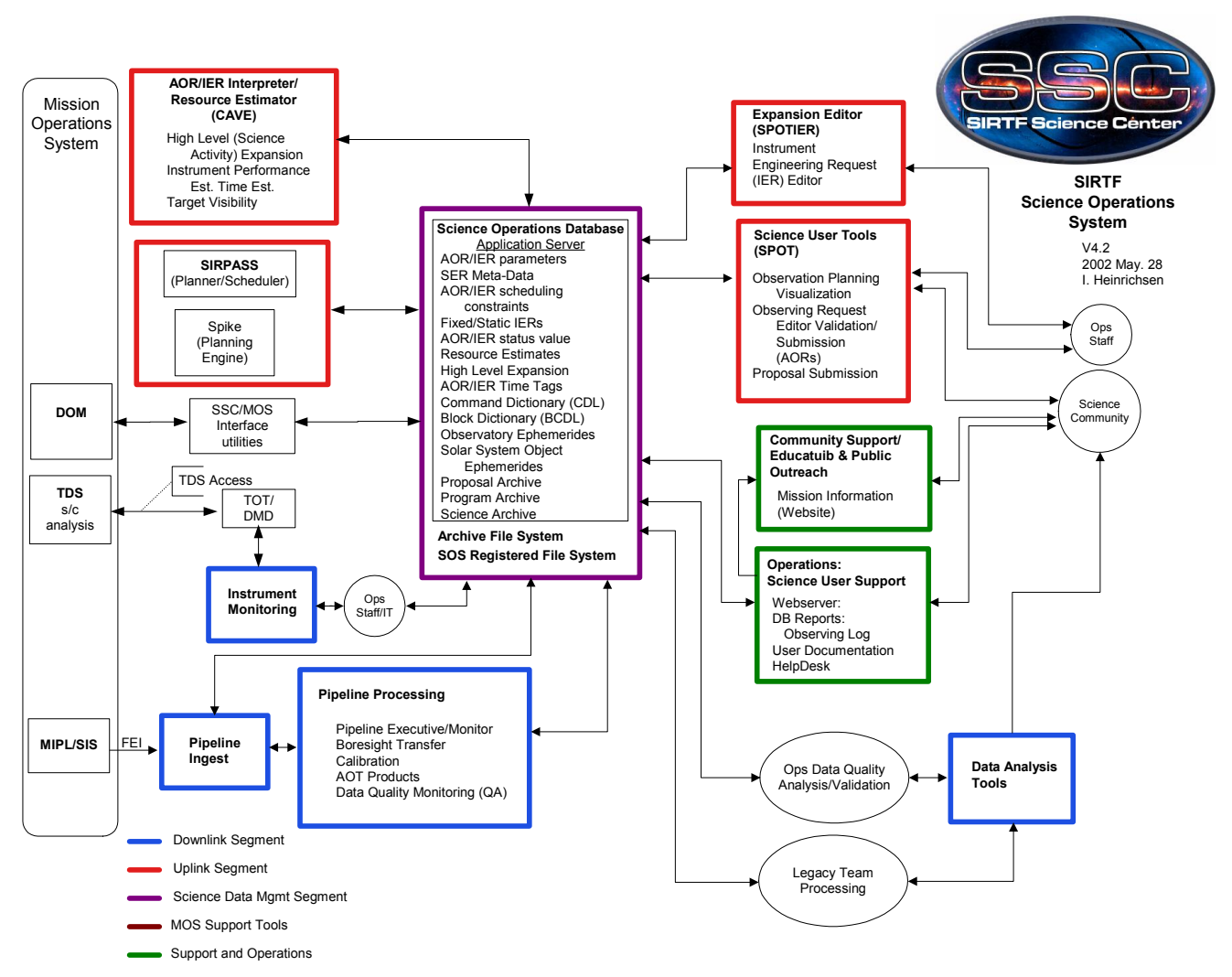

Fig. 2: Science Operations System

The Science User Tools consist of a variety of Java-based software elements that are downloaded to the user's PC or workstation. Users can maintain a catalog of observation targets on their home machines. They can select from one of seven observing modes across the three payload instruments, and input observation parameters to generate an AOR (Astronomical Observation Request). Users can build a library of desired observations on their home systems, and then, when completed, download the final set of observation requests to the SSC. Observers maintain a local database of candidate observations on their own computer systems, and then make a final selection and download the files to the SSC as part of the proposal submittal process. Data visualization tools can be used to overlay templates of specific observation requests, graphically on images requested from a variety of archival databases, including the 2MASS image atlas recently released. .

The AOR/IER Expansion Editor is used to expand each individual AOR (or Instrument Engineering Request IER's) into spacecraft and instrument commands. The user is provided with the resource estimate for each observation following expansion of the AOR or IER into a command sequence.

The Science Observations Data Base (SODB) is used at the SSC to hold all information submitted by the community, and will eventually encompass the project archival data bases as well. Command expansions are transferred to the flight operations systems at JPL for execution via transfer from the SODB to JPL operational data systems. Downlink data is transferred to the SODB by the JPL flight operations system when data are acquired by the Observatory and transferred to the JPL ground data support systems from the Deep Space Network. The SODB now contains approximately 10,000 AOR's from the GTO and Legacy Teams. 
The SSC pipeline processing is an automated process that is initiated when data requested by the uplink planning system are delivered to the SODB. The SSC is currently designing and implementing the pipeline systems with collaboration by the instrument Principal Investigators and their support teams.

\section{SCIENCE USER TOOLS}

The tools used by observers to plan their observations have been under development at the SSC for over three years, and in active use by the science community for over two years. The major tool used by observers is the SIRTF Planning and Observation Tool (SPOT) ${ }^{1}$. SPOT provides access to all operating modes of the three SIRTF instruments, and provides a variety of capabilities used by scientists to plan observations using SIRTF capabilities.

The logical unit of information throughout the SOS is the Astronomical Observing Request. Observers utilize a set of templates (Astronomical Observation Templates or AOT's) for each instrument that allows them to request a specific observation to be acquired with a specific mode of instrument observation. There are seven planned AOT's for SIRTF, with four of the modes operational at launch. An example of one of the AOT's is shown in Figure 3, the Spectral Energy Distribution (SED) mode for the Multiband Imaging Photometer for SIRTF (MIPS) instrument. The user enters desired exposure time, and other parameters, and the software expands the AOR to the command level and returns resource estimates as shown. The resource estimates are computed using current models for spacecraft slew and settling times which are updated periodically based on Observatory test data. The models will be updated periodically during operations based on experience.

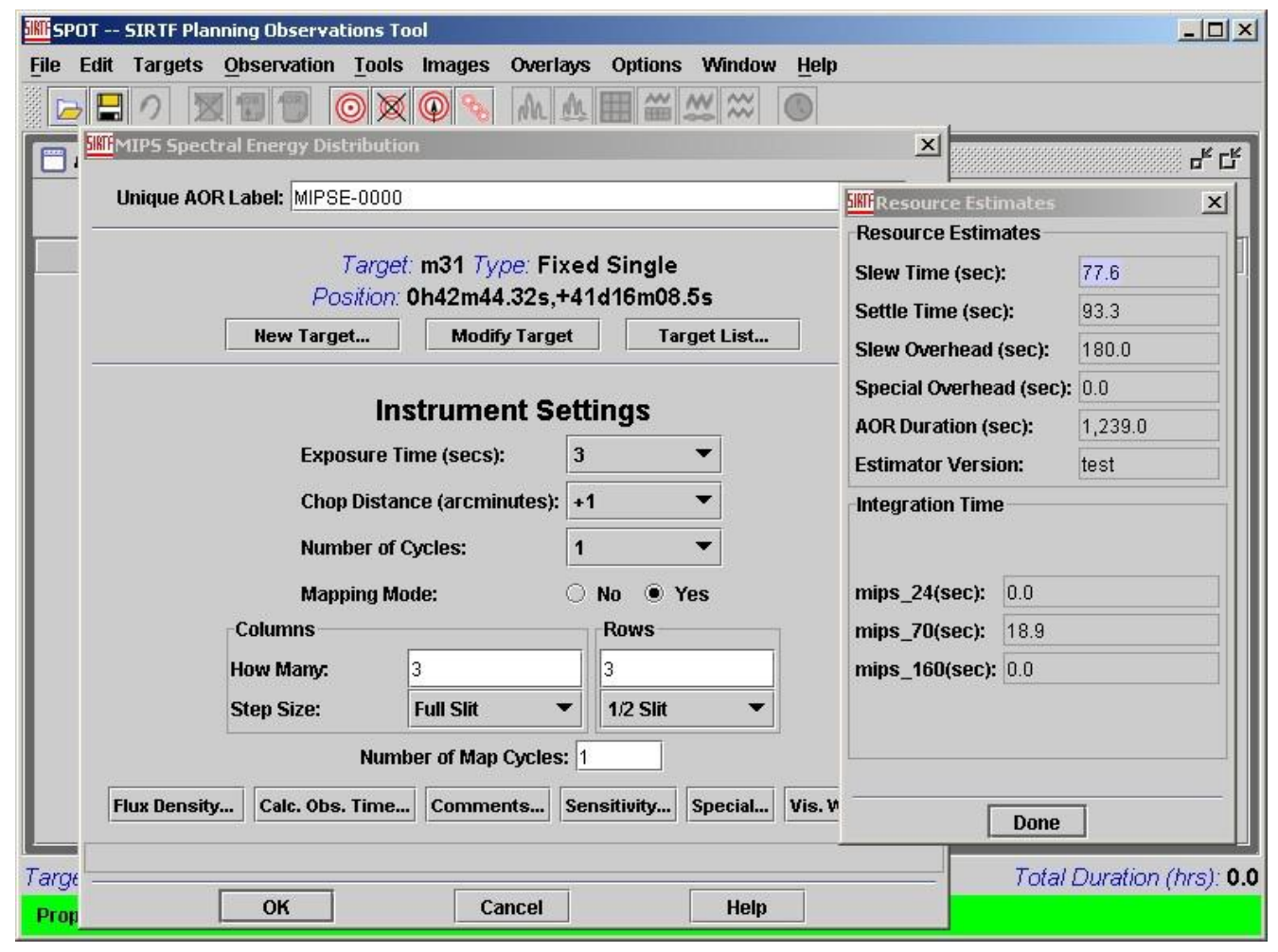

Fig. 3: Astronomical Observing Request

\footnotetext{
${ }^{1}$ Roby, T., Writing a Large Application in the Real World on the Java Foundation Class/Swing API, Java One conference, 2002, http://servlet.java.sun.com/javaone/resources/content/sf2002/conf/sessions/pdfs/1703.pdf
} 
Users can maintain a database on their local computer of desired targets, and candidate AOR's that comprise their observation proposal. A final editing by the user is performed prior to submittal of the command expansion files generated by each AOR as part of the observing proposal. Figure 4 shows a listing of AOR's from all 3 instruments, for several different targets. The list of targets being maintained by the user is also shown in the "List of Targets" window. SPOT utilizes the NASA Extragalactic Data Base (NED) and other resources for name resolution of astronomical targets, providing the position for targets with names that reside in those external databases.

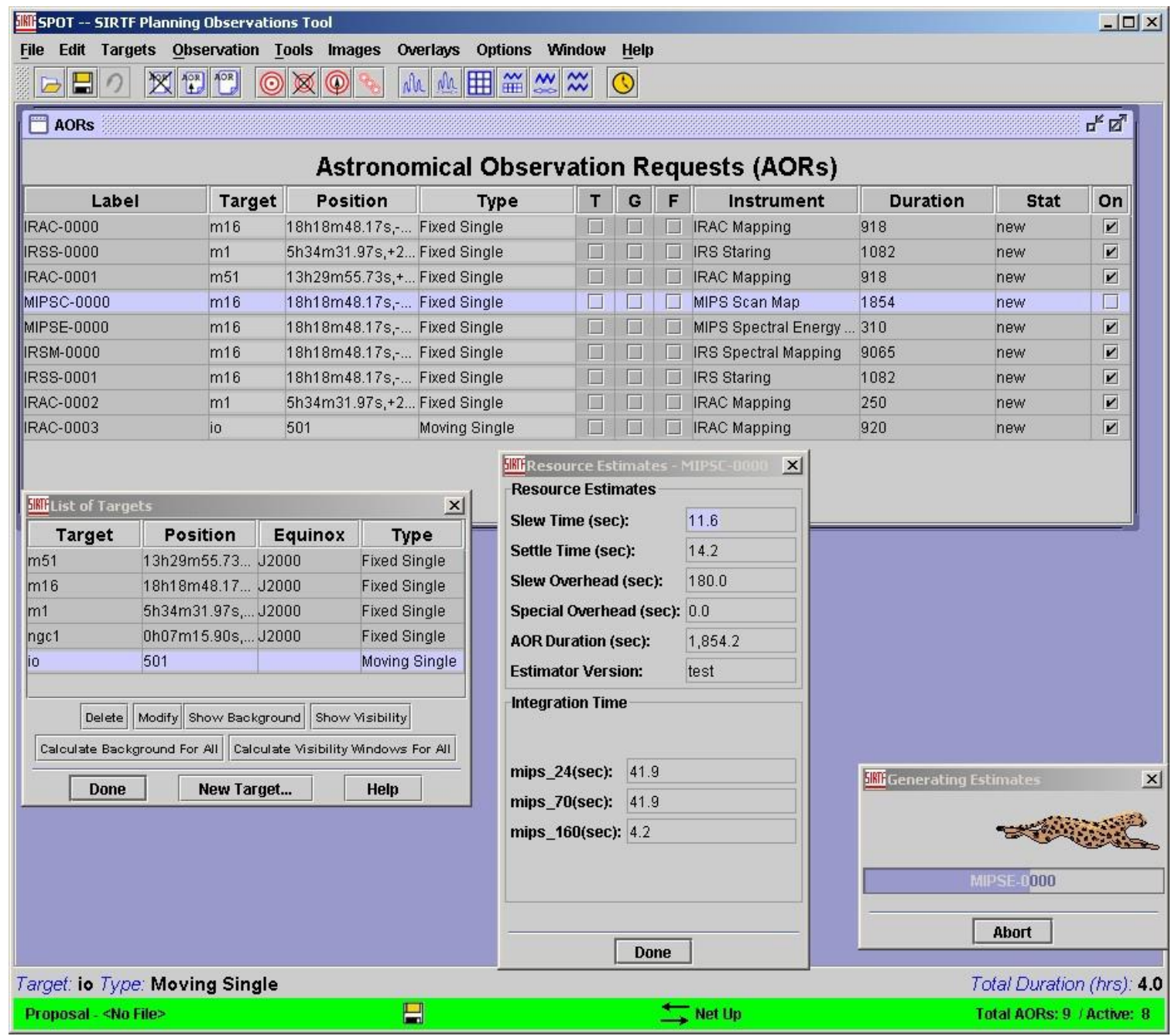

Fig. 4:Local Storage of AOR's and Targets

Figure 5 shows an example of the data visualization support capabilities available within SPOT. The footprint of an IRAC (Infrared Array Camera) AOR has been overlaid on images from two different data sources that serve as reference images. The Digital Sky Survey (DSS) image is in the background, and an image from the MSX mission has been overlaid on the DSS image. SPOT has been used to register the reference images into a single visualization display and to overlay the footprint of the SIRTF IRAC observations on the reference images. 


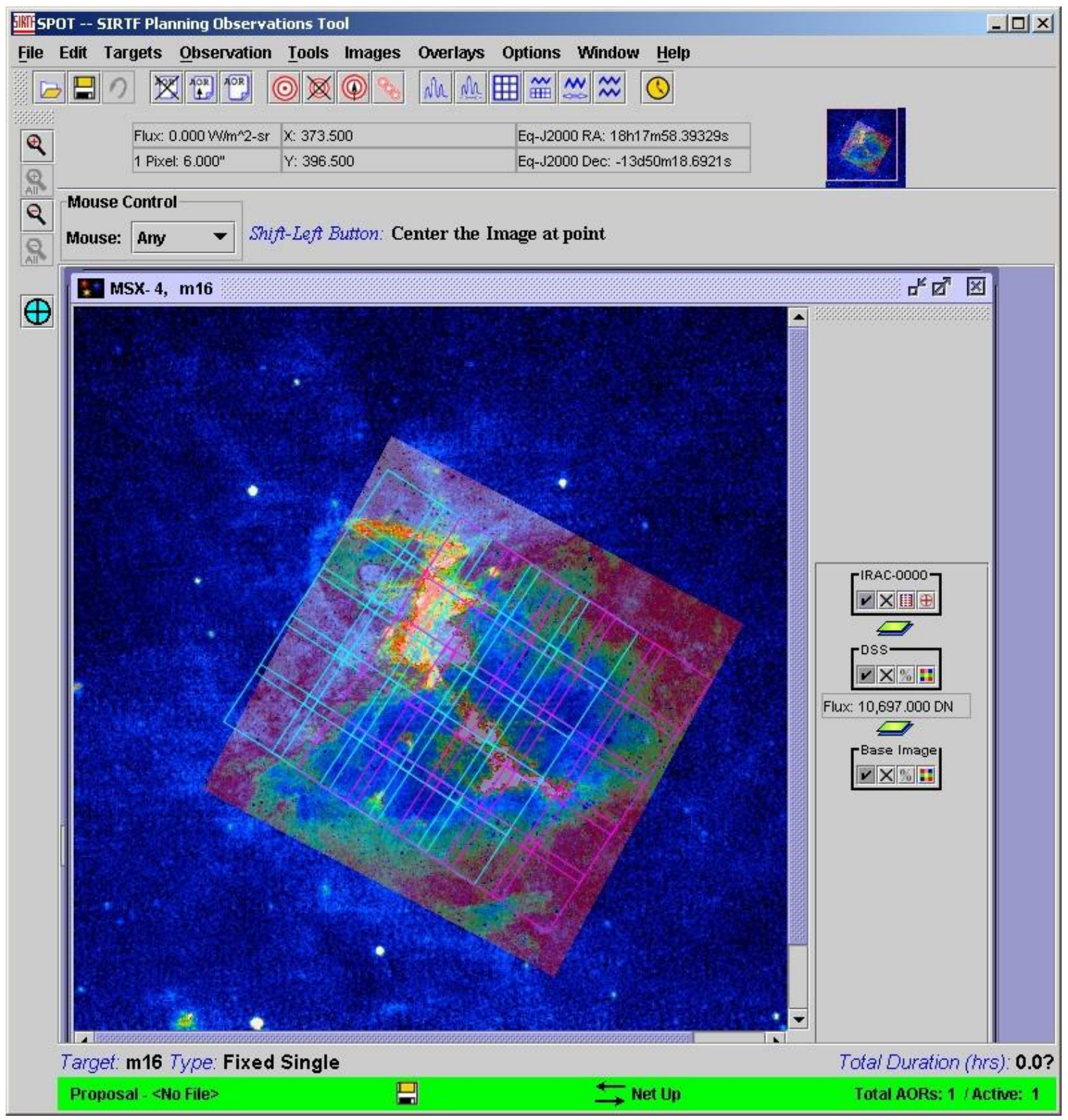

Fig. 5: Data Visualization of a SIRTF observation

SPOT has been used by the GTO and Legacy scientists to provide their observation programs to the SSC for over two years. The Science Observations Database (SODB) at the SSC now contains over 10,000 AOR's, representing the detailed observation requests from the GTO's, Legacy Teams, and the 100 hour First Look Survey to be performed immediately after In-Orbit Checkout. These AOR's are being used to do detailed planning of the first year of science observations.

\section{OBSERVATORY PLANNING AND SCHEDULING}

The SSC uses mission planning and scheduling software developed for SIRTF, using the SPIKE system from the Space Telescope Science Institute with additional software developed at the SSC. The planning and scheduling system is called SIRPASS (SIRTF Planning and Scheduling System). SIRPASS is designed to perform both long range planning and short term scheduling through sequence packaging. The process is shown in Figure 6. 


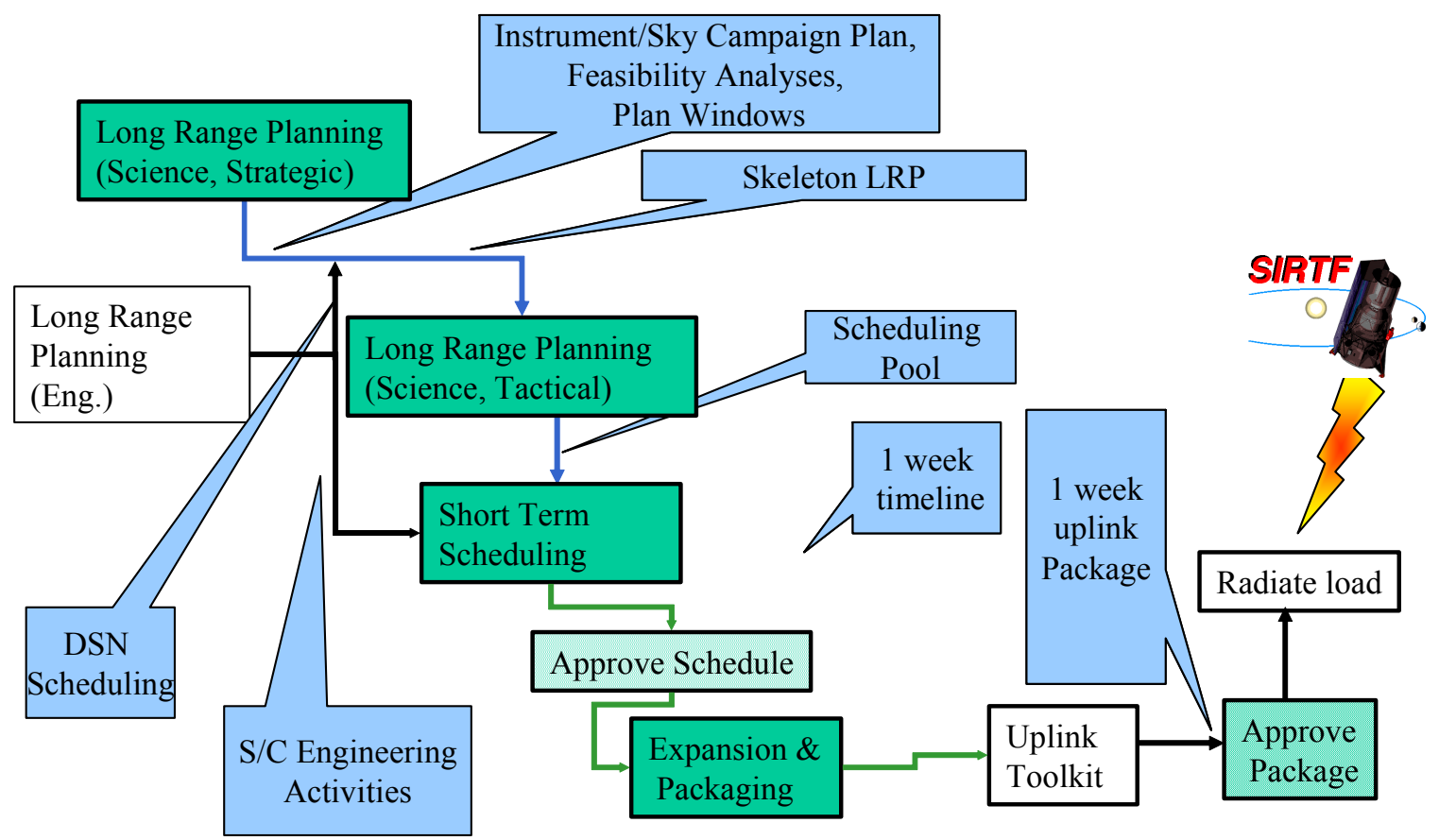

Fig. 6: Observatory Planning and Scheduling Process

The long range planning function is performed by analyzing the AOR's stored in the SODB, instrument engineering requests (IER's) provided by the three instrument support teams at the SSC, and information regarding scheduling requirements for Spacecraft Engineering provided by the SIRTF flight engineering team. Long range planning (LRP) provides a long range plan that allocates planning windows for each instrument by analyzing target visibility as a function of time and multiple constraints associated with the AOR's, IER's and spacecraft engineering events. AOR constraints can include requests by observers to acquire data in contiguous periods of time, in a specific order, or other constraints. IER's and spacecraft engineering events can be specified as fixed time activities (e.g. data downlinks) or have other constraints associated with them. SIRPASS produces a Baseline Instrument Campaign (BIC) for an extended period of time (e.g. a year) that allocates planning windows for instrument observations. A "Proof of Concept (POC)" BIC produced from an early version of the mission SODB at the SSC that contains a total of 10,000 AOR's is shown in Figure 7. The plot shows AOR's for all three SIRTF instruments distributed across instrument campaigns through the first year of operations, with campaign durations of from 3 to over 10 days.

Once the BIC is approved by the SSC Director, short term scheduling is then performed on a week by week basis. SIRPASS utilizes the SPIKE software to extract individual AOR's and attempt to schedule them with minimal wasted time, incorporating the constraints associated with each AOR along with other constraints associated with the IER's and spacecraft activities. A weekly schedule is produced and approved by the SSC Director, at which point sequence files are produced and transferred to the Mission Sequencing Team (MST) at JPL. The MST then packages the sequences and transmits the uplink load to the Observatory. The SSC utilizes metadata for the spacecraft activities during the scheduling process (basic data regarding the duration and timing of the activities). The MST integrates the actual command data for the spacecraft activities into the sequence as part of the packaging process. 
POC 2 Baseline Instrument Campaign

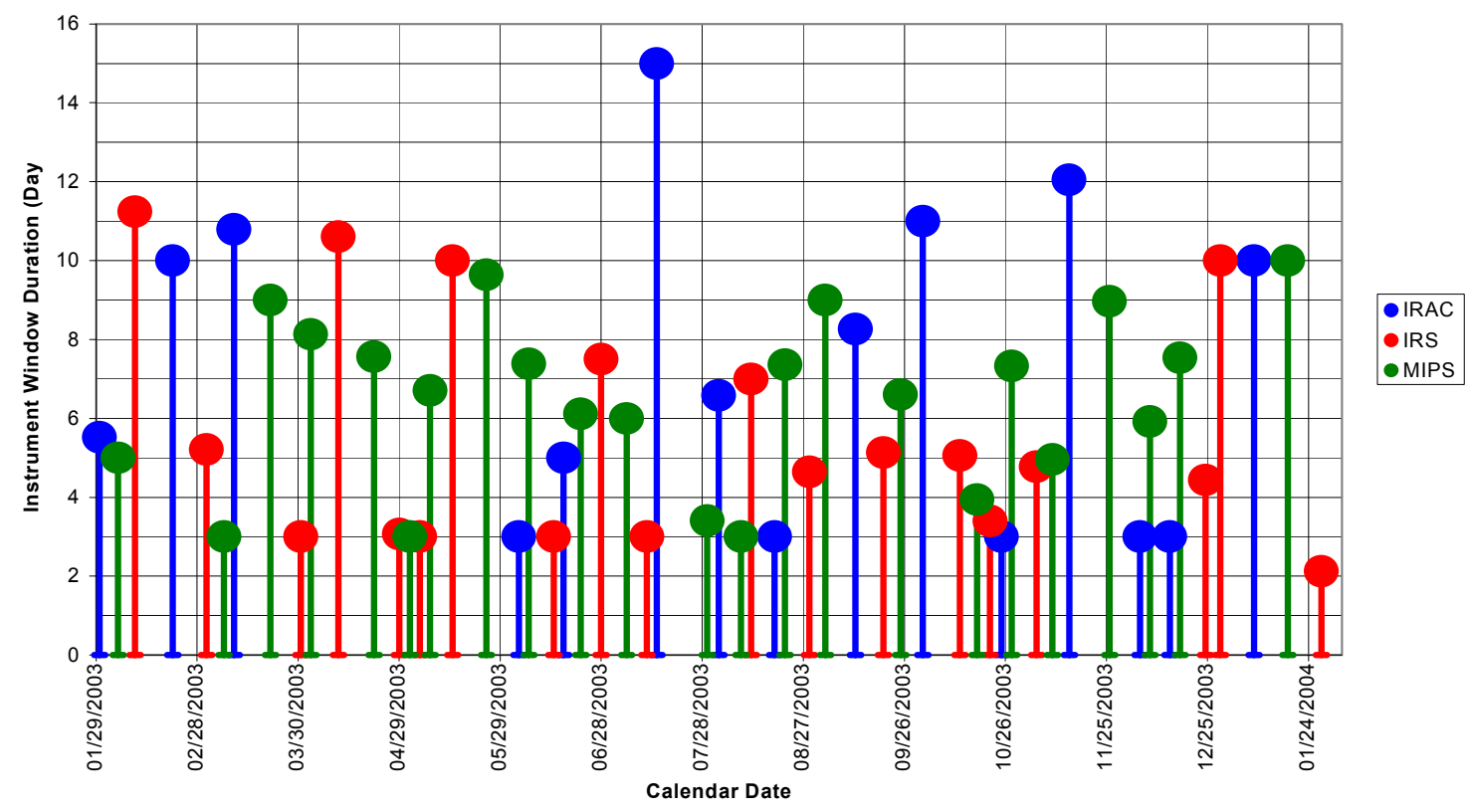

Fig. 7: The Proof of Concept Baseline Instrument Campaign

One result from a weekly scheduling exercise is shown in Figure 8. Time percentages are shown for one week of observations for science observations and instrument calibration activities, spacecraft calibration and data downlink, slews between targets, and gaps with no activities scheduled. Prelaunch planning of the first year's activities is now in process, using updated information from the GTO and Legacy Teams.

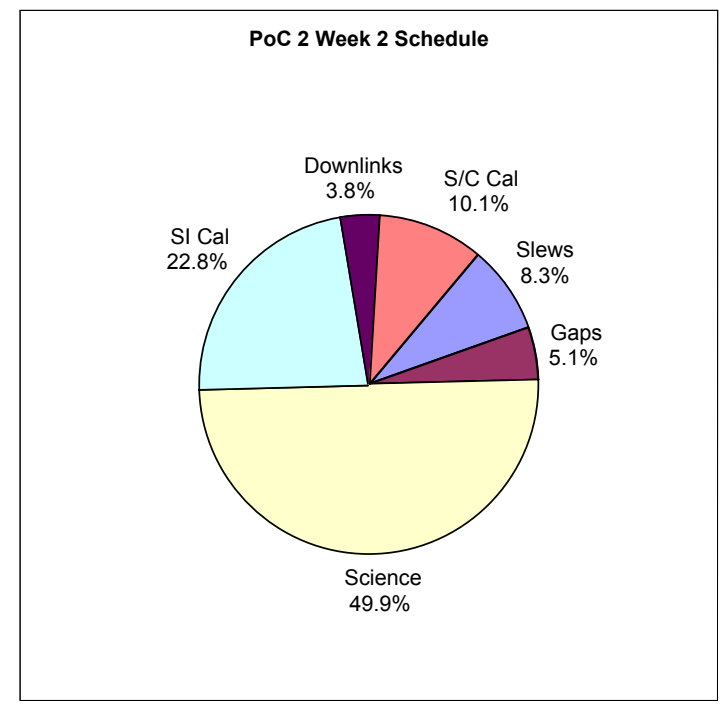

Fig. 8: Allocation of time in Week 2 of the scheduled produced as part of the Proof of Concept activity 


\section{DATA PIPELINES}

SIRTF is planning a sixty day In Orbit Checkout (IOC) period following launch, followed by an additional 30 day Science Verification (SV) phase. IOC is intended to be the time period in which the project demonstrates that the Observatory meets its Level 1 functional requirements. The SV phase will be used to certify the AOT's for each instrument, including any required updates to the AOT templates and data pipelines needed to accommodate in-flight instrument and Observatory performance.

There are multiple pipelines planned as part of downlink processing of the science instrument data and production of the archival data products. Data from each AOR will be used to produce a Basic Calibrated Data product (BCD) that will be placed into the public archive of science products. In addition, other threads through the pipeline will support instrument calibration activities and long term instrument performance monitoring activities at the SSC.

Each of the Instrument Principal Investigators and their teams have worked with SSC staff to define the processing to be performed for each mode of instrument operation. Preliminary pipelines for each instrument will be operational at launch, with updates planned during IOC and SV phases to accommodate actual inflight instrument and Observatory performance One thread for the Infrared Array Camera is shown in Figure 9.

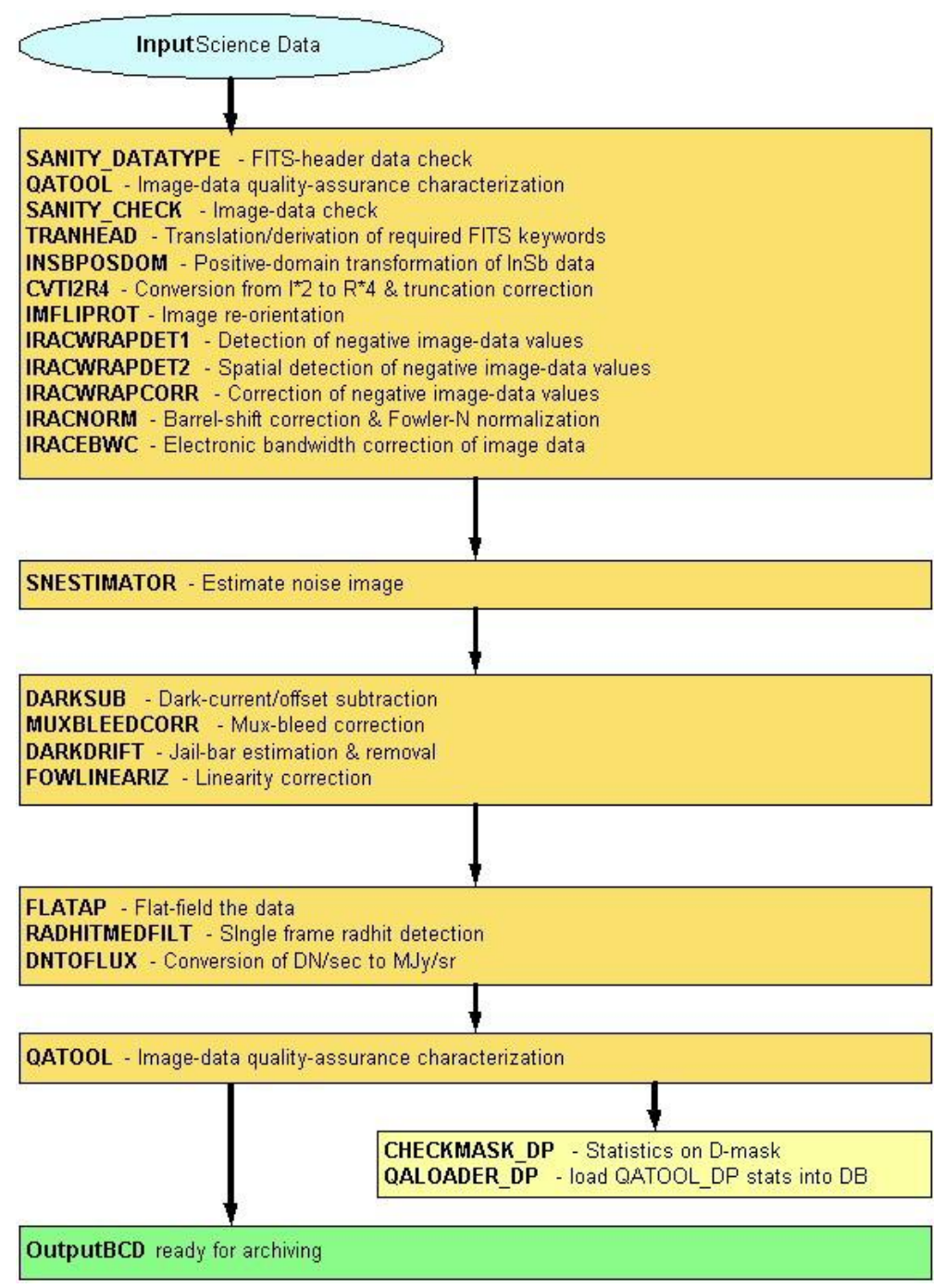

Fig. 9:Pipeline data flow for production of an IRAC BCD Product 
The raw data and BCD product for a simulated observation of NGC4589 by the Infrared Array Camera are shown in Figures 10 and 11. Figure 10 illustrates a simulation of the actual instrument, including instrument effects observed in preflight instrument testing.

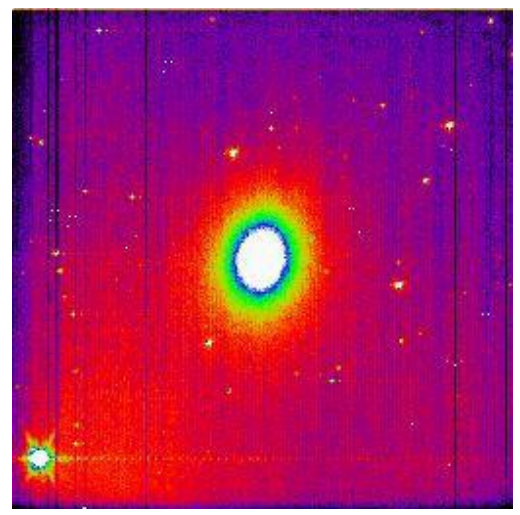

Fig. 10: Simulated raw IRAC image of NGC 4589

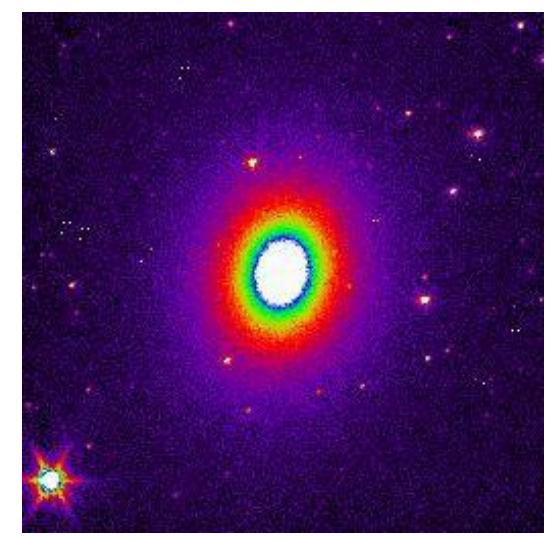

Fig. 11: BCD product generated by SSC pipeline, using process from Figure 9 on raw image in Figure 10

Note the effects of flat fielding and removal of instrument induced artifacts in Figure 11. Each AOT mode for each instrument will be processed in an automated manner by the SSC pipelines following receipt of the complete data set for each AOR. Data quality assessment pipelines will be run as well, and flag any problems in the processing for analysis by SSC operations teams.

SSC has been involved with pipeline development and processing of instrument test data for several years, working in collaboration with the PI's and the teams responsible for instrument development and characterization. The SSC will assume responsibility for the instrument operations and long term performance trending of the science instruments following completion of the SV phase of the mission.

\section{CONCLUSION}

The SSC is nearing completion of the prelaunch version of the Science Operations System, following almost four years of development. The system now comprises over 1 Million lines of code. Formal testing of the system has been performed at the system and subsystem level at fixed delivery points throughout the development cycle. In the past six months, sequences produced by the SOS have been sent to JPL, packaged by the JPL flight operations system and executed on the Observatory flight hardware or on spacecraft and instrument simulator systems. Downlink products generated by flight hardware and instrument simulators have been processed at the SSC, and SSC staff have monitored engineering data 
from these tests in real time utilizing data systems that will be used during flight operations. The final readiness review for SIRTF flight operations will occur late in 2002, prior to the launch in early 2003.

\section{ACKNOWLEDGEMENTS}

The current state of maturity of the Science Operations System is due to the efforts of the outstanding staff of the SIRTF Science Center in designing, developing, implementing, testing and operating the systems that will be used to support SIRTF operations, and their efforts in supporting the external scientific community. The cooperation received from the three instrument Principal Investigators, Dr. Giovanni Fazio, Dr. James Houck, and Dr. George Rieke, is gratefully acknowledged. Thanks also to the SSC Director, Dr. B. Thomas Soifer, and Deputy Director, Dr. George Helou, to Project Manager Dave Gallagher and Dr. Michael Werner, Project Scientist at JPL, for their support. The end to end system level testing of the SSC developed capabilities integrated with the JPL-developed mission operations system is under direction of Bob Wilson, the SIRTF Mission Operations System Manager at JPL This work was performed at the California Institute of Technology under contract to the National Aeronautics and Space Administration. 